\title{
Evaluation of the technical condition of medium-sized boilers
}

\author{
Pavel Lošák ${ }^{1, *}$, Tomáš Létal ${ }^{1}$, Jiři Buzík ${ }^{1}$, Martin $\mathrm{Nad}^{11}$ \\ ${ }^{1}$ Brno University of Technology, Faculty of Mechanical Engineering, Institute of Process \\ Engineering, Technická 2, 61669 Brno, Czech Republic
}

\begin{abstract}
The recent trend in the steam and electricity production has been both to increase the efficiency of the facility and to keep tightening legislation concerning emission limits. The lifetime of energy equipment is greatly influenced by the operating temperature, pressure and operating characteristics. The new conditions lead the operator to more often changes of these parameters, which has negative influence to the facility in terms of service life. Precise knowledge of the facility being operated and the ability to predict the residual life of its key parts in time is therefore necessary. A new methodology for determining the residual life and evaluating problematic situations of medium size boilers was developed at Brno University of Technology. Its approaches and advantages will be presented in this paper. The methodology provides the user with approaches for the lifetime evaluation of an equipment as a whole, based on detailed knowledge of the equipment being investigated and the ongoing damage. Additionally, if the equipment is continuously evaluated, it is possible to extend the inspection interval or to achieve a significantly higher lifetime of the entire equipment, thereby reducing the economic cost. If defined criteria are met, the methodology also allows inclusion of FEM and CFD simulations for achieving higher relevance of the results.
\end{abstract}

Keywords: lifetime evaluation, boiler damage

\section{Introduction}

Safe operation of the equipment is one of the key requirements for technical device and must be taken into account both when designing the equipment and operating it. Inappropriate device design may lead to public safety threats as well as considerable economic losses. Together with safety, the economy of operation also plays an important role. Each part of a device has a certain lifetime, after which it is necessary to replace the part or to repair it in such a way as to avoid unplanned shutdown of the device. Appropriate design, operation and maintenance, together with appropriate and timely repairs, can extend the time the device will be able to operate safely.

With the development of science and technology, there is still a growing knowledge and refinement of the methods enabling meeting of these requirements. However, with the development of technology, there is also an increasing demand for materials. Operating

\footnotetext{
* Corresponding author: losak.p@fme.vutbr.cz

Reviewers: Jan Krmela, Ireneusz Malujda
} 
temperatures and pressures increase as well as the need for operative changes in production that may unfavorably affect the device in many ways. It is therefore necessary to thoroughly understand the principles of damage and on the basis of these, carry out expert analyses describing the behavior of the investigated unit. Still, the current level of science and technology is not so high that it can capture the complexity of processes and take into account all the parameters that affect the behavior of the device. It is therefore important to apply appropriate simplifying assumptions leading to results without significant deviation from the reality under consideration.

There are a number of degradation mechanisms detrimental to boiler operation. Presented article focuses on the field of steam boilers and methodology for the evaluation of their technical condition. This methodology deals with a comprehensive approach contributing to safe and economically acceptable operation of the equipment. It also includes an appendix containing procedures for device diagnostics. Methodology deals with assessment of fatigue, corrosion, high temperature oxidation and erosion. In order to prevent accidents and their associated health and economic risks, it is necessary to carefully investigate and evaluate the degradation mechanisms. These mechanisms are becoming increasingly important today. Based on efforts to increase the share of renewable energy use in power generation, a significant part of electricity is produced today by solar and wind power plants. This new phenomenon leads to frequent instabilities in the power grid, which is due to probabilistic nature of these alternative sources. Suddenly, it is necessary to compensate for these instability in the power grid. These requirements may also have an impact on the operation of steam boilers, where previously almost monotonous operation transformed to operation with significant cyclical changes.

In some European Union countries, support for alternative energy sources is also provided by the government offering financial benefits to those who are willing to compensate the emerging power grid instabilities. Here the precise knowledge of damage mechanisms can help in deciding whether it is financially advantageous to continue increasing electricity production despite the accelerated damage of the equipment.

The recent trend is the tightening of emission limits and associated fines for those who are unable to meet these limits. Reducing emissions is closely linked to the need to increase efficiency. This increase in efficiency is linked to the need for higher pressures and higher temperatures in the process. Current steam parameters can be at "ultra-super critical" levels, which corresponds approximately to $600{ }^{\circ} \mathrm{C}$ and 305 bar. The next generation of ultra-super critical level II will have temperatures even higher at $700{ }^{\circ} \mathrm{C}$. Selection of materials that are able to withstand such harsh conditions for prolonged time is seriously limited. Therefore, there is still ongoing research and development of new materials able to meet these challenges. However, the problem may occur when the increased operating conditions are applied to materials that have not been designed for these parameters at the design stage. This is the case, for example, when the operator is forced to reduce the emissions of existing plant and will therefore increase steam parameters. In such cases, there is a potential problem with damaging the oxide layers. Although oxide layer formation is common, elevated temperatures or rapid temperature changes may lead to a massive breakdown of the cohesiveness of the oxide layers. That may result in blockage of pipe throughput, turbine blade erosion, and other complications.

All these changes lead to an increased need for accurate monitoring and evaluation of the device. The presented article is devoted to the development of a methodology for the on-line and off-line assessment of damage to equipment. 


\section{Motivation}

One of the commonly performed activities at the Institute of Process Engineering, where this methodology has been developed, is the implementation of so-called troubleshooting analyses of equipment from the energy industry. In these activities, we repeatedly encountered a critical lack of backgrounds for performing appropriate analyses to identify the causes of the problem situation, their effect on the equipment and the subsequent proposal for corrective measures. Therefore, a methodology was developed that would summarized the necessary data to perform precise simulations of the equipment operation and allowing to propose accurate and effective corrective measures in case of a failure. Moreover to allow plan changes of operating parameters in so-called "on-line" diagnostics. Such an assessment would allow the operator to manage the residual life of the plant and monitor the effects of the operating states for residual life.

The knowledge of the operating history and other documented documents is of vital importance for the evaluation of the equipment. Without these backgrounds, which is common case for many devices, troubleshooting is complicated and requires a number of simplifications. Although the results do not fully describe the reality under examination, they may still provide valuable information as they present the only available information that is relevant to decisions about corrective measures. Even in this case, the methodology can be a valuable guideline for performing the analyses, even though under simplified assumptions.

\section{Evaluation methodology}

The presented methodology is divided into categories that affect or are necessary to determine residual service life and troubleshooting. The methodology provides a set of requirements for monitoring, evaluating and storing data so that qualified lifetime management can be performed. Also, verification of the methodology is possible after it is used by the operator. The methodology consists of verified parts, such as calculations according to standards, for which no further verification is presumably required (although still possible) and the parts that are considered with the verification. Verification is based on comparison of the calculation results with the current state of the device according to inspections of the equipment. In the case of a disagreement between the calculated life expectancy and the actual condition of the equipment, the methodology further considers the possibility of applying corrective measures.

The methodology also focuses on establishing criteria that would make it more transparent and allow for the independent replication of finite element (FEM) and flow simulation (CFD) numeric analyses, which in certain cases presents an undisputed advantage in the device diagnostics, search for causes of damage and design of corrective measures.

\subsection{Main parts}

The main parts of the methodology that summarize the complex approach to device evaluation are as follows: Design calculations, Documented quality, Device monitoring, Device Diagnostics, Device status evaluation and Troubleshooting. Each chapter contains a list of documents that are relevant to that section. The more precisely will the evaluation be according to these modules, the more accurately can the life of the equipment be determined and consequently the associated activities such as revision plans optimized. In the event of unplanned damage to the equipment, a qualified assessment of the problem 
situation and the design of an appropriate corrective measures can be made in accordance with the proposed methodology.

\subsubsection{Design calculations}

Designing equipment in accordance with standards is also the basis for determining residual service life. Design is tied to the use of valid regulations and safety requirements according to the applicable regulations of the given area. An important part of the design is also the risk analysis according to the relevant regulations. Design calculations must include, in particular, the determination of the planned lifetime of the installation and also take unavoidable additional loads into account.

\subsubsection{Documented quality}

For the life management system, respectively for the ability to perform qualified assessments of residual lifetime or to deal with unexpected problem situations, the documents recording complete and accurate information about the activities performed are crucial. The documented quality can be divided into the areas of production, operation and exceptional cases. In the area of the manufacturing part it is necessary to keep all relevant documents related to this area which document the fabrication of the equipment and will be useful in the future in solving problematic situations. Area of operation will include the operational records of the facility, including inspections, along with information about the shutdowns and startups of the facility. Some of the exceptional cases will include data relevant to the situation, e.g. information about specific degradation of material characteristics discovered during an inspection.

\subsubsection{Equipment monitoring}

Equipment monitoring will be designed based on the most probable and detrimental degradation mechanisms that may occur under expected normal as well as exceptional operation conditions. Parameters that will be monitored with the appropriate degradation mechanism are as follows:

Static load:

- pressure

- temperature

- actual dimensions

- material parameters

Variable loads:

- temperature and pressure gradient

- actual dimensions

- material Parameters

- numbers of load cycles according to their character (cold, warm hot)

Additional loads due to the nature of the operation:

- media temperatures

- wall surface temperatures

- forces in anchorages

- moves

Load by operating medium - corrosion, erosion, oxidation:

- supply water composition

- fuel composition 


\subsubsection{Equipment diagnostic}

The diagnosis of the equipment will be carried out according to the criteria given by the relevant standards. Other methods may be used to diagnose the device, when the suitability for use is demonstrated. Diagnosis can be done with commercially available software or using in-house procedures. Part of the presented methodology is a description of usable procedures for device diagnostics in a given area, which can be used for development of an evaluation software. These procedures are included in the appendix to this methodology. Diagnosis will be done on-line, with the damaging trend determined from the continuous monitoring as well as manual evaluation of the damage found during the inspections.

\subsubsection{Equipment condition evaluation}

The damaging trend estimated by on-line diagnostics will be compared to the original design lifetime. The evaluation of the residual life and condition of the equipment will be further extended by a lifetime correction based on the actual state of the equipment ascertained during carried out inspections. Consideration will also be given to changes in service life due to repairs and changes in the design of the equipment.

In case when the damage accumulation factor exceeds allowable limit, it is possible to modify the approach to assessing the equipment according to its current state and to set a new lifetime plan, on the basis of which the maintenance and inspection plan will be modified.

There may also be situations where the calculated cumulative damage factor is lower than the actual damage found during the inspection. In this case, it is necessary to introduce the correction factor of the damage accumulation factor until the cause of this discrepancy has been identified and corrective measures (modification of the operating conditions or assumptions for calculating the accumulation of damage) have been made.

\subsubsection{Troubleshooting}

If unexpected failures occur, troubleshooting will be performed. Detailed records of the production, operating and other stages can be used to identify the cause of the damage and to propose corrective measures together with a modification of the life plan. For this activity, it is advisable to use the services of expert organizations with modern computing tools.

\section{Evaluation methods}

The first stage will include visual inspection of the problem situation and the establishment of a procedure leading to appropriate corrective actions. These activities will consist of:

- material analysis

- detail analysis of operating conditions

- visual checks to detect abnormal function of parts of the device (e.g. poorly adjusted burners, inappropriate distribution of emission-reducing additives)

- checking the dimensions

- a detailed strength calculation that takes into account the actual state of the equipment and the subsequent identification of the factors that caused the damage

- detailed calculation of media flow and analysis of their impact on the problem situation

- combined strength and flow analysis and identification of problem factors 


\section{Strength calculation methods}

Heat calculation, flow simulation and subsequent strength calculation will be performed for a particular part of the plant. If possible, analytical analysis will be performed. Other methods, for example, finite element method or computational fluid dynamics method can be used if analytical solution is not available.

\section{Strength calculation by FEM}

For strength calculation, it is recommended to consider the requirements of standards, for example according to ČSN EN 13445-3 [1]. The standard permits several useful methods. One method is "design by analysis". Two approaches to the solution can be used, the first is a stress categorisation route. An elastic calculation is performed, which is then evaluated by assigning the respective stresses to the categories and then comparing these stresses with the permitted stresses according to the requirements of the standard. This method yields conservative results that may overestimate expected damage in some cases. For further refinement the second method called the direct route can be used. The direct route method is the calculation performed according to the limit states. This method checks each possible structural failure separately. The design checks considered are:

- control of the structure to gross plastic deformation,

- progressive plastic deformation,

- loss of stability,

- fatigue,

- checking static equilibrium of the structure.

Further refinement of residual life estimates may be also achieved using specific non-linear analyses. These can provide accurate determination of time-dependent stress and strain results together with eventual multi-axial behaviour. These calculations help to locate dangerous areas in the structure, and consequently aid choice of sensor type and placement for continuous monitoring as well as refine revision plans. It is also possible to predict the growth rate of identified cracks and to assess their acceptability for further operation.

\section{Flow calculations using CFDs}

In some cases, where non-uniform boundary conditions cannot be determined using conventional methods due to complex flow, they may be established using CFD simulation. Useful boundary conditions from CFD include pressures, forces, heat transfer coefficients and temperatures. In case of non-uniform thermal field, thermal analysis may be also performed before final structural analysis. CFD analysis consists of the following steps:

\section{Preparation and simplification of geometry}

In this step, either 3D geometry is taken from the design solution or newly created according to the drawing documentation. If $3 \mathrm{D}$ geometry is assumed, it is first necessary to create an inverse volume - that is, fill the internal volume of the device with fluid in the areas where the CFD simulation will be performed, remove / suppress the volume of solid walls of the device, and then proceed to refine and simplify the geometry created. Trimming is often necessary because there are details in the design drawings that do not need to be considered in terms of flow.

The result should be "multibody part" geometry. All volumes should be coupled in one part to ensure a conformal mesh at the interfaces of the individual volumes. Basically, it's a good idea to avoid using non-conformal mesh that requires interpolation of results between meshes.

\section{Creating a mesh}

The computing mesh (grid) is a basic precondition for a correct and convergent calculation. The basic requirement is to adequately describe the areas of high gradients of each 
modelled variable. One of the important gradients that need to be correctly described is the speed and temperature boundary layers of the walls. Here it is necessary to take into account the used turbulent models or wall functions as well and to correctly select the size of the first cell in the wall according to the recommended dimensionless parameter $y+$. If we want to achieve the most accurate resolution, at least 10 cells perpendicular to the wall with the first cell of size $y+<1$ in the boundary layer area must be used.

\section{Setting boundary conditions and selecting physical and numerical models}

First, it is necessary to determine which physical models will be included in the calculation. For preliminary analysis, it is often advantageous to neglect the thermal expansion of fluids if there are no significant changes in the temperature in the calculation area. Likewise, it is possible in certain cases to neglect the compressibility of the fluids under small changes of pressure. Another options to consider is radiation modelling. It always depends on the temperature ratios in the computing domain and the properties of the fluid. In general, it is advantageous to perform the first calculation in a simplified form and then proceed to include more complex physical models.

One of the key aspects of flow modelling is the choice of a turbulent model when the flow is in turbulent regime.

According to the selected setting, it is necessary to proceed to determining and specifying boundary conditions. Their choice has a major impact on the results of the calculation. It should be based on measured real-time data, previous numerical simulations, or in the case of new technology from design parameters.

The choice of numerical models should be derived from the type of mesh and physical models. However, in practice, it is necessary to use higher-order discretization for all equations. For the convergence evaluation, it is always necessary to evaluate the stability of the physical quantities (e.g. velocity or temperature) in addition to so-called residuals. It is also always necessary to evaluate the total weight and energy balance.

\section{Evaluation of results and export of data}

The evaluation should always include verification of the quality and suitability of the computing mesh. It is suitable to use 2D colour contours of individual physical quantities. For these contours, the colour range showed should represent the full range that is present in the displayed area. If, for some reason, the scale is modified, it must be stated and explained in the text.

Data transfer for eventual FEM analysis is possible in several ways, depending on the software used. Ideal is to use direct interconnection within one software, which guarantees minimal distortion of the data being transferred. In the case of data transfer between different software, attention should be paid to the way of interpolation.

\section{Conclusion}

In the present article, a methodology which was designed to increase the reliability and safety of steam boiler operation was presented. The emphasis and main contribution of the methodology is to characterize the documents that are created during all phases of the steam boiler lifetime from its design to the end of its lifetime and may be required for lifetime management. The methodology is composed of the design part, the operating part, the diagnostic part and the part of the evaluation of unexpected states, where the use of modern computational methods such as FEM and CFD is promoted. Together with the implementation of these methods in the equipment evaluation, there is a need for precise specification of the use of these methods. It is necessary to adhere to certain rules and to document important steps when performing these analyses so that it is further possible to replicate these analyses by an independent person. For the purpose of replication, the results 
of the analyses as well as input data need to be documented. For the diagnostics of the device, whether on-line or off-line, the methodology includes an annex proposing methods for determining the proportion of individual degrading mechanisms to the overall damage of the examined unit.

The authors gratefully acknowledge financial support provided by Technology Agency of the Czech Republic within the research project No. TE02000236 "Waste-to-Energy (WtE) Competence Centre"

\section{References}

1. Český normalizační institut, ČESKÁ TECHNICKÁ NORMA, Netopené tlakové nádoby. ČSN EN 13445. 2015. 